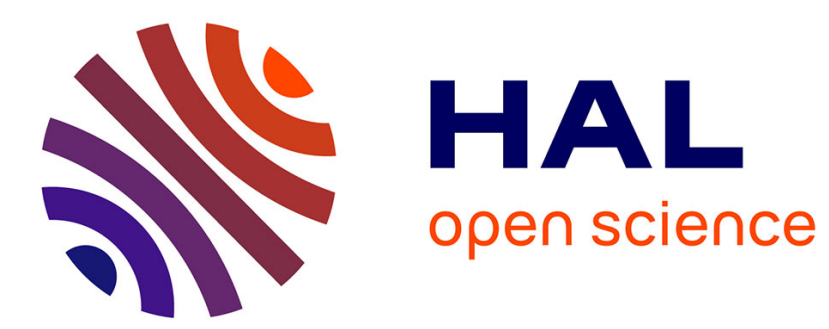

\title{
Sur les traces des disparus au Mexique
}

Sabrina Melenotte

\section{To cite this version:}

Sabrina Melenotte. Sur les traces des disparus au Mexique. Ethnologie française, 2020, $\mathrm{N}^{\circ} 178$ (2), pp.345-360. 10.3917/ethn.202.0345 . hal-02571204

\section{HAL Id: hal-02571204 https://hal.science/hal-02571204}

Submitted on 19 May 2020

HAL is a multi-disciplinary open access archive for the deposit and dissemination of scientific research documents, whether they are published or not. The documents may come from teaching and research institutions in France or abroad, or from public or private research centers.
L'archive ouverte pluridisciplinaire HAL, est destinée au dépôt et à la diffusion de documents scientifiques de niveau recherche, publiés ou non, émanant des établissements d'enseignement et de recherche français ou étrangers, des laboratoires publics ou privés. 


\title{
Sur les traces des disparus au Mexique
}

\author{
Sabrina Melenotte
}

IRD-URMIS / FMSH-ANR SoV

sabrinamelenotte@ird.fr

Presses Universitaires de France | «thnologie française »2020/2 N 178 | pages

345 à 360

ISSN 0046-2616

https://www.cairn.info/revue-ethnologie-francaise-2020-2-page-345.htm

\section{RÉSUMÉ}

Le Mexique connaît depuis douze ans des violences extrêmes et massives modifiant en profondeur le traitement politique, culturel et social des disparus et des morts en masse. Devenue récemment une priorité dans l'agenda politique, la disparition, forcée ou non, reste une énigme pour les vivants hantés par l'incertitude et les fantômes. Face à l'inaction de l'État, les familles des victimes font « justice par elles-mêmes » et conjurent cette malemort par deux principales actions : «lire la terre » pour en faire émerger des fosses clandestines et «lire le cadavre » pour rendre une identité à ces morts qui dérangent. À partir d'une description ethnographique d'un dimanche de recherche de fosses clandestines menées en 2016 avec des familles du collectif «Les autres disparus » à Iguala, dans l'État du Guerrero, cet article démontre à quel point suivre les traces des disparus signifie davantage que mener de simples fouilles : les recherches de fosses clandestines concentrent l'opération de restauration d'un ordre matériel et symbolique face au puzzle macabre laissé par les violences politiques et criminelles. Elles constituent de nouveaux rituels modernes et réintroduisent du sens et du sacré là où les traces des crimes sont camouflées.

Mots-clés : Mexique. Disparitions. Traces. Fosses clandestines. Cadavres.

\section{- Introduction}

Le Mexique connaît depuis douze ans des violences extrêmes et massives alors même que le pays a connu une transition démocratique trente ans plus tôt. La militarisation de la société mexicaine liée à la lutte officielle contre le narcotrafic, impulsée en 2007 par l'ancien président de la République Felipe Calderón Hinojosa, a paradoxalement augmenté les homicides ainsi queles activités illégales du narcotrafic [Escalante, 2011 ; Musset, 2015]. Après une alternance entre le parti de droite (Parti d'action nationale, PAN) et le parti historique (Parti révolutionnaire institutionnel, PRI), les chiffres officiels font aujourd'hui état de plus de 235000 morts et 62000 disparus. Andrés Manuel López Obrador, élu nouveau président de la République mexicaine en juillet 2018, cherche à rompre avec les gouvernements précédents. Le nouveau régime annonce des transformations institutionnelles importantes : la «Loi générale en matière de disparition forcée de personnes, de disparition commise par des particuliers et du système national de recherche de disparus », votée en novembre 2017 sous le gouvernement précédent, peine encore à être appliquée ; une Commission de vérité et de justice promet de résoudre l'affaire des 43 étudiants de l'école rurale d'Ayotzinapa, disparus dans des conditions obscures en 2014 dans l'État du Guerrero, et reprend l'enquête à zéro ; un Système national de recherche de disparus ainsi que des instituts d'expertise se mettent aujourd'hui en place à travers une nouvelle Commission pour répondre à la crise humanitaire et légiste, mais sans le personnel compétent ni les ressources suffisantes. Le nouveau gouvernement prend donc des mesures au niveau national pour les dizaines de milliers de disparus et leurs proches, mais il creuse en même temps le sillon d'une amnistie pour les petits producteurs de drogue et crée une Garde nationale plaçant la police sous mandat unique et direct de l'armée, ce qui interroge sur la rupture réelle avec les mesures sécuritaires passées pour affronter la grave crise de légitimité de l'État mexicain. L'urgence à traiter les disparus et morts en masse ouvre de nouvelles perspectives sur le traitement politique, culturel et social de la mort et de la disparition dans les prochaines années. Une réelle «transition funéraire »a 


aujourd'hui lieu au Mexique qui s'accélère sous l'impulsion de l'agenda politique mexicain qui fait du phénomène de la disparition une priorité.

Cette histoire contemporaine marque indubitablement les esprits et les imaginaires de tous les Mexicains : l'expérience de la perte et de la mort en masse représente une rupture d'intelligibilité et d'entendement pour les familles. Pourtant, j'aimerais plutôt prendre le contrepied d'une interprétation paralysante de la disparition, et montrer que des proches des victimes, mortes ou disparues, partent sur leurs traces dans plusieurs États du pays et créent de nouveaux rituels permettant de dépasser les enjeux politiques, sociaux et culturels d'une société brutalisée et d'une nation bouleversée : en cherchant leurs disparus, les familles matérialisent l'absence et recréent un ordre social et symbolique dans une humanité rompue. La disparition forcée n'est donc pas seulement un symptôme des violences politiques et criminelles. Elle soulève des interrogations sur la manière dont les vivants vivent avec des fantômes et recréent du sens dans ce puzzle macabre.

La disparition d'une personne est une énigme en soi : elle impose la présence de l'absent parmi les vivants, et les hante. Se dessine alors un paysage fantomatique autour d'une vie ou d'une mort incertaines. Ces dernières années, l'un des moyens trouvés par les familles pour résoudre l'énigme et conjurer la malemort est de faire parler la terre d'où émergent régulièrement des morts qui dérangent, dans des fosses communes et clandestines. En la prenant comme sujet anthropologique, j'aimerais montrer que la disparition génère une vie politique, sociale et culturelle d'une intense fécondité. «Faire apparaître » [Claverie, 2011] les corps, morts, disparus ou enfouis, est la condition sine qua non pour aider le deuil des familles, la réparation des victimes, l'exigence de vérité sur le passé et de justice pénale et sociale. Face au déni d'État, les recherches de fosses clandestines deviennent alors davantage que de simples fouilles : elles concentrent l'opération, matérielle et symbolique, d'un potentiel retour à l'ordre par le deuil des familles de disparus, qui font justice " par elles-mêmes », voire se substituent à l'État. Je mettrai donc l'accent sur le caractère performatif des recherches de fosses pour montrer qu'elles constituent des nouveaux rituels modernes visant à restaurer un ordre menacé et réintroduire du sens et du sacré là où les " officiants de la violence » cherchent à camoufler le crime de multiples manières.
Après mes recherches doctorales sur le conflit armé dans l'État du Chiapas, j'ai débuté une nouvelle enquête dans l'État du Guerrero ${ }^{1}$ à l'été 2016. Ces premières observations, en partie relatées ici, parlent d'un temps déjà révolu, où les premières recherches de fosses clandestines se faisaient de manière autogérée par les familles et n'étaient pas aussi soumises aux protocoles d'aujourd'hui. Ces recherches de fosses se sont désormais multipliées dans le pays, elles ont été progressivement encadrées par des autorités judiciaires et des experts. Ces descriptions consignent donc l'histoire récente des disparitions en accomplissant un arrêt sur images qui me permet de développer deux moments précis de ce que j'appelle, non sans paradoxe, l' «ethnographie des disparitions » : la localisation des fosses puis l'identification des corps. Ces moments permettent d'appréhender la trace sous ses aspects matériels et symboliques de deux façons. Lapremière est celle de la trace comme marque, empreinte, externe et visible dans l'environnement géographique, la trace que l'on suit tel un chasseur ou que l'on exhume, tel un archéologue, pour en faire une piste, un indice. Les traces laissées par les violences politiques ou criminelles se matérialisent d'abord dans l'environnement géographique : la terre recèle des signes matériels et symboliques qui dessinent des «paysages de la mort », ponctués de références et de traces qui deviennent pour les familles des signes et des indices que «quelque chose » s'est passé «ici », «à un moment donné ». Puis les violences marquent les cadavres que l'on retrouve. La seconde dimension relève ainsi d'une anthropologie de la trace dans le cadre de violences extrêmes et massives [Sémelin, 2005; Anstett et Jurgenson, 2009] : la trace y devient une preuve matérielle des pratiques répressives, institutionnelles ou criminelles. Elle est une inscription, une signature de l'exécuteur de la violence. La disparition devient donc un acte d'énonciation qui engendre des pratiques communicationnelles singulières et qui pousse à lire les paysages de la mort et les cadavres.

Je décrirai ici un dimanche de recherches de fosses clandestines dans les montagnes aux abords de la ville d'Iguala. Le point le plus frappant réside certainement dans les techniques de repérage de ces fosses qui rappellent fortement le geste originel du chasseur analysant les empreintes de sa proie. Les familles développent des connaissances relevant du paradigme indiciaire analysé par Carlo Ginzburg [1980] pour élaborer une compréhension de « ce qui s'est réellement passé » : voir, sentir, enregistrer, interpréter, classifier 
les « traces infinitésimales » est une opération millénaire consistant à déchiffrer et interpréter des traces d'événements qui sont des signes a priori anodins pour établir un savoir expérimental à partir d'une réalité, elle, non directement expérimentable ${ }^{2}$. Lors des recherches de fosses, c'est un peu comme si les analyses, comparaisons et classifications des familles relevaient du paradigme cynégétique sur les empreintes des violences ; et comme si celles des experts légistes prenaient appui sur le paradigme divinatoire, par une nouvelle forme d'écriture microscopique qu'est la génétique, pour lire dans les corps l'identité d'un anonyme et réécrire le futur d'une nation dontl'humanité est aujourd'hui fragmentée. Ensemble, ces deux savoirs consolident le paradigme indiciaire, toutes les séquences de la recherche de disparus visant à créer un récit issu du déchiffrement et de l'analyse des traces.

La description des recherches de fosses met en évidence un ensemble d'acteurs " non-étatiques », tels que les familles, les défenseurs des droits humains et l'Église catholique progressiste, et d'autres acteurs institutionnels, les experts légistes et les autorités judiciaires mobilisés pour prendre en charge «le destin des corps morts » [Anstett et Dreyfus, 2012], chacun produisant des savoirs et savoir-faire entre science, vérité et politique. L'épreuve des corps, les outils et techniques employés, ainsi que les méthodes d'observation expérimentale qui se développent lors des recherches dans les montagnes, grâce au rôle crucial et ancien de la perception, notamment de la vue [Dulong, 1998 ; Didi-Huberman, 2004], aujourd'hui bousculée par le tournant médico-légal [Anstett et Dreyfus, 2015] qui considère de plus en plus les traces devenues imperceptibles, liées à l'usage de la génétique dans l'identification des cadavres.

\section{- Disparaître dans le Guerrero}

Toute disparition, forcée ou non, massive ou non, est une énigme pour une société car elle contient dans sa définition même l'effacement de la trace attestant que « quelque chose » s'est passé. On pourrait même dire que parler de disparition suppose d'emblée un paradoxe : les disparus n'existent pas en tant que tels. Ce qui existe, ce sont des êtres et des corps sans noms, souvent à l'état de restes, des assassins, souvent libres, et des vivants marqués par une brutale et soudaine incertitude. Pourtant, par l'évocation de leur absence, les disparus matérialisent un lien social et politique inscrit dans une violence quotidienne marquée par l'invisibilité, le silence, le non-dit, voire le tabou. Dans le Mexique contemporain, l'effacement peut être l'enfouissement ou la dissolution de la trace du crime par des mises à mort d'une extrême cruauté. Les techniques de camouflage du crime alternent avec l'exhibition de corps suppliciés visant à engendrer de la terreur.

En juin et juillet 2016, j'ai débuté une nouvelle enquête dans l'État du Guerrero situé au sud-ouest du pays. Aujourd'hui, c'est l'un des États les plus marginalisés et l'un des premiers producteurs de marihuana et de pavot, surtout dans les régions reculées des montagnes de la Sierra Madre. Berceau de l'indépendance du pays en $1821^{3}$, la municipalité d'Iguala est devenue une étape obligée pour les Cartels, dont la nature et le nombre se sont considérablement modifiés ces dernières années ${ }^{4}$. C'est aussi dans cette municipalité qu'une manifestation étudiante a été réprimée en septembre 2014, faisant 6 morts, 25 blessés et 43 disparus, tous étudiants de l'école rurale d'Ayotzinapa. Cet événement obscur a révélé la crise politique nationale sévère dans le pays, à savoir la corruption, voire la collusion entre le crime organisé, les autorités politiques (le maire d'Iguala) et les forces de l'ordre, allant des polices municipales à l'armée. Il a surtout été à l'origine de fortes mobilisations qui ont abouti, en novembre 2017, à la réforme juridique reconnaissant le statut de disparition forcée 5 .

Les disparitions forcées sont anciennes dans les cas chilien, argentin, uruguayen, espagnol, bosniaque, cambodgien ou même nazi. Elles le sont également, du bassin méditerranéen au Mexique contemporain en passant par la Colombie, ce qui fait dire à Gabriel Gatti [2017] que la disparition est devenue une catégorie qui possède une vie sociale en soi et qu'elle circule aujourd'hui dans des sphères transnationales, au point de créer de nouveaux espaces de la souffrance contemporaine. Si la Méditerranée peut être lue comme une fosse de milliers de déplacés et réfugiés, de même au Mexique, le leitmotiv des familles de disparus estque « le pays est une énorme fosse ». La métaphore de l'espace-temps géologique singulier de la fosse redessine à la fois des «paysages médico-légaux » [Cyr, 2014] et des paysages mentaux, intériorisés, où l'épreuve de la disparition obsède les vivants et oriente leur regard à la recherche de signes qui pourraient émerger de la terre. Ces « paysages de la mort» sont 
d'abord soumis aux conditions géographiques et climatiques, mais aussi aux représentations régionales de la mort et de la disparition. L'ethnographie est donc plus à même de discerner la catégorie générique de « disparition forcée » en l'ancrant dans un «paysage de la mort » singulier qui se décline au niveau régional.

$\mathrm{Au}$ Mexique, les disparitions, forcées ou non, recouvrent des réalités hétérogènes. Les mères ontété les premières à s'organiser dans le nord du pays, premier champ de bataille de la guerre contre le narcotrafic. Dans les États de Coahuila, de Basse-Californie, de Sinaloa, de Nuevo León, les violences liées à la lutte contre le narcotrafic ont laissé des fragments de corps très difficiles à identifier car les méthodes d'exécution ont été particulièrement destructrices. Dans le Sinaloa, les mères de disparus se font appeler les «rastreadoras » (celles qui suivent les traces) et marchent sans relâche depuis le début de la guerre contre le narcotrafic. À Coahuila, elles ont poussé à un plan d'exhumation au niveau fédéral bien avant la réforme nationale de la Loi sur la disparition forcée. Au centre du pays, dans le Morelos, les recherches de disparus se concentrent depuis 2015 sur des fosses « communes », c'est-à-dire légales, situées dans des cimetières avec des exhumations et des identifications de cadavres réalisées dans un cadre extrêmement contrôlé. Pourtant, de nombreuses irrégularités apparaissent de la part des autorités, qui traînent les pieds.

Dans le Guerrero, le profil des disparus récents est homogène : $80 \%$ sont de jeunes hommes pauvres, métis ou indiens. Dans les régions les plus reculées de la Sierra Madre où les paysans indiens produisent le pavot et le cannabis, à Chilapa par exemple, l'impunité est quotidienne : non seulement les homicides et les disparitions sont plus élevés, mais les personnes ne déposent pas de plaintes, par peur des représailles. À Iguala, la population est essentiellement métisse et pauvre. Les morts violentes y sont quotidiennes : alors que $\mathrm{j}$ 'arrivais pour la première fois chez Xitlali qui m'accueillait chez elle, elle m'annonce immédiatement que la semaine précédant mon arrivée, 18 personnes au total avaient été assassinées, dont 4 décapitées. Le lendemain, le neveu de Doña Sandra que je rencontrais pour la première fois venait de se suicider avec de l'herbicide car son petit ami faisait partie de ces morts. J'assistais le surlendemain à ses funérailles.

À Iguala, les familles connaissent souvent et côtoient malgré elles les bourreaux de leur disparu : Doña Sandra croise régulièrement dans la rue le puissant sicaire du coin, père de celui qui a d'abord séquestré sa fille durant des mois, avant de la « vendre » car « elle était jolie », la faisant donc disparaître. Doña Caritina quant à elle habite en face d'un soldat qui a fait disparaître de nombreux corps, dont celui de son fils ; Don Rogelio connaît le député fédéral lié à l'enlèvement de son fils. Les motifs de leur disparition sont toujours obscurs, mais les familles sont convaincues del'innocence de leurs jeunes fils enlevés injustement et ce n'est d'ailleurs pas le centre de leurs préoccupations : « nous ne sommes pas là pour les juger, mais pour les

retrouver ». Les autorités judiciaires, au contraire, pensent que ces jeunes étaient pour les trois quarts impliqués dans des activités liées au narcotrafic, soit en tant qu'informateurs (appelés halcones, faucons), soit en

tant qu'exécutants de petites tâches (prélever les quotas, récupérer de l'argent, transporter de la drogue ou d'autres produits, etc.) dans la chaîne du narcotrafic. Ces visions opposées ont des conséquences herméneutiques importantes, puisque les familles rendent coupables l'État de ne pas mener l'enquête, tandis que les autorités interprètent les crimes comme des règlements de compte entre narcotrafiquants et les disparitions comme méritées d'une manière ou d'une autre.

Avant 2014, rares étaient les familles qui osaient porter plainte suite à la disparition de leurs proches. Vivant dans un climat de peur, l'omerta l'emportait sur le sentiment d'injustice, les familles préféraient se taire par peur des représailles des bandes criminelles organisées, mais aussi en raison d'une extrême méfiance envers les autorités qui, considéraient-elles, étaient les mêmes qui les faisaient disparaître. Le scandale qui suivit la disparition des 43 étudiants d'Ayotzinapa a agi comme un véritable déclencheur pour ces familles de disparus dans l'ensemble du pays. Rien qu'à Iguala, plus de 350 familles ont déposé leurs plaintes en quelques mois, après s'être aperçues de la condition commune qui les unissait. C'est ainsi qu'est née l'organisation «Los Otrosdesaparecidos» (les Autres disparus), grâce à 1 'appui d'une police communautaire indigène ${ }^{6}$ qui a organisé les premiers rassemblements au centre d'Iguala, constitué les premiers dossiers et encadré les premières recherches de fosses clandestines. 


\section{- Lire la terre}

\section{Sur les traces des disparus}

Dès la fin de l'année 2014, une fois les plaintes déposées, les premières recherches de fosses clandestines ont été réalisées sans autorités judiciaires ni experts. Ceux-ci ne sont arrivés qu'en mai 2016, après que les familles aient cherché seules. Au début, les familles cherchaient tous les jours, et progressivement, seulement les dimanches. J'ai donc suivi les familles qui partaient sur les traces des disparus autour d'Iguala, à un moment où leurs recherches étaient déjà avancées et qu'elles trouvaient moins de fosses. J'ai observé la course folle de ces familles de disparus s'improvisant chasseurs et détectives et soumettant leurs corps à rude épreuve. L'obsession de la disparition de leurs proches les poussaient à marcher des journées entières, sans relâche, dans les montagnes, malgré l'âge de certains et des conditions climatiques parfois adverses liées à une chaleur écrasante ou des chemins difficiles. Le jeûne et la mobilisation aiguë des sens (vue, odorat, toucher) lors des recherches octroient une place prépondérante au corps qui marche et qui devient lui aussi un véritable outil pour tracer les morts. La nature environnante est alors davantage qu'un paysage à contempler : elle relate des violences passées et contient les derniers moments de centaines de vies. Les montagnes qui entourent Iguala hébergent donc des secrets qui jaillissent des entrailles de la terre et qui créent une cartographie souterraine d'histoires individuelles qui peuvent refaire surface. À mon arrivée l'été 2016, les familles de disparus avaient déjà «lu la terre » au point d'avoir découvert 150 corps dans les alentours d'Iguala en un an et demi de recherche. Devenues «chercheuses de fosses », elles avaient réussi l'exploit de faire parler des paysages de la mort où trouver une fosse clandestine revenait à chercher une aiguille dans une botte de foin. Elles s'exposaient également au risque d'être vues, ou de croiser toute personne impliquée dans le narcotrafic, les zones de recherches étant identiques aux zones de passage des criminels. La semaine suivant mon départ, des menaces de mort directes envers les familles ont été proférées sur les réseaux sociaux par des individus se réclamant du cartel Les Rouges (Los Rojos), instillant une peur puissante parmi les familles organisées dont beaucoup ont fui Iguala un certain temps.
Le départ se faisait le dimanche devant l'église de San Gerardo. Depuis peu, une effigie de la Vierge de Guadalupe, offerte par le curé jésuite de Cuajimalpa (Morelos), accompagnait les familles pendantl'expédition. Les premières recherches de fosses clandestines par les familles ont été le fruit de leur auto-organisation, sans autorités judiciaires ni experts, tout au plus un journaliste local les suivait. À Taxco, ville coloniale et touristique non loin d'Iguala devenue prospère pour l'exploitation de ses mines d'argent, la sœur d'un disparu a un jour tiré sur une botte qui dépassait de la terre, convaincue qu'il s'agissait de celle de son frère. Cette image dans la presse locale et régionale a fait grande impression car elle rendait flagrante l'absence d'autorités et de procédures légales. Ce scandale médiatique a obligé le parquet fédéral (Procuraduría General de la República, PGR $)^{7}$ à attester de son effort pour chercher les disparus, en reprenant la direction des opérations et faire du groupe de recherche des « Autres disparus d'Iguala » un projet pilote, supplantant le travail des autorités fédérées [Fiscalías] généralement en charge des enquêtes ${ }^{8}$.

Les autorités judiciaires encadrent donc les recherches de fosses clandestines depuis le début de l'année 2016. Mais aujourd'hui encore, elles dépendent totalement des familles qui ont une connaissance poussée des montagnes et des relations sociales. Elles les suivent et ont besoin d'elles à la fois pour obtenir des informations, s'orienter et se repérer dans les montagnes. Leur présence manque cruellement de discrétion dans une zone où tout se voit et se sait : les deux pickups blancs des familles sont escortés par 4 à 6 autres véhicules bleus et blancs de la police fédérale, parfois de l'armée, un rouge de l'équipe du procureur, une camionnette blanche d'une unité canine et de 4 à 5 experts légistes qui tournent chaque semaine (criminologue, photographe, vidéaste, anthropologue physique), parfois aussi de la commission nationale des droits humains (Illustration 1). Ces démonstrations de force des représentants officiels ne sont toutefois pas suivies d'un grand zèle dans les actes. Elles établissent des relations ambivalentes, voire de franche méfiance, de la part des familles avec les autorités judiciaires : lors de ma première recherche avec les familles, cellesci sont arrivées avec deux heures de retard; la semaine suivante, elles ont déplacé l'horaire de rendez-vous d'une heure et demie, sur demande de la Marine, alors affairée par des manifestations importantes des enseignants partout dans le pays. Puis, lors des recherches, les autorités judiciaires, les polices et la Marine 
refusent d'entrer sur les terrains privés sans mandat d'arrêt, à l'inverse des familles qui n'hésitent pas à franchir les clôtures des terrains privés pour explorer. $\mathrm{Ce}$ respect de la loi freine, voire empêche des possibilités d'exploration de fosses parfois indiquées ou repérées par les familles sur les terrains privés des caciques ou des sicaires locaux. Ici, l'invocation de la loi et du protocole par les représentants de l'État contraste fortement avec la négligence, voire l'omission fréquente de certaines procédures, comme de ne pas accompagner ou prolonger les pistes des familles, ne pas excaver sérieusement ou le faire rapidement, ne pas produire de rapports systématiques de toutes les recherches.

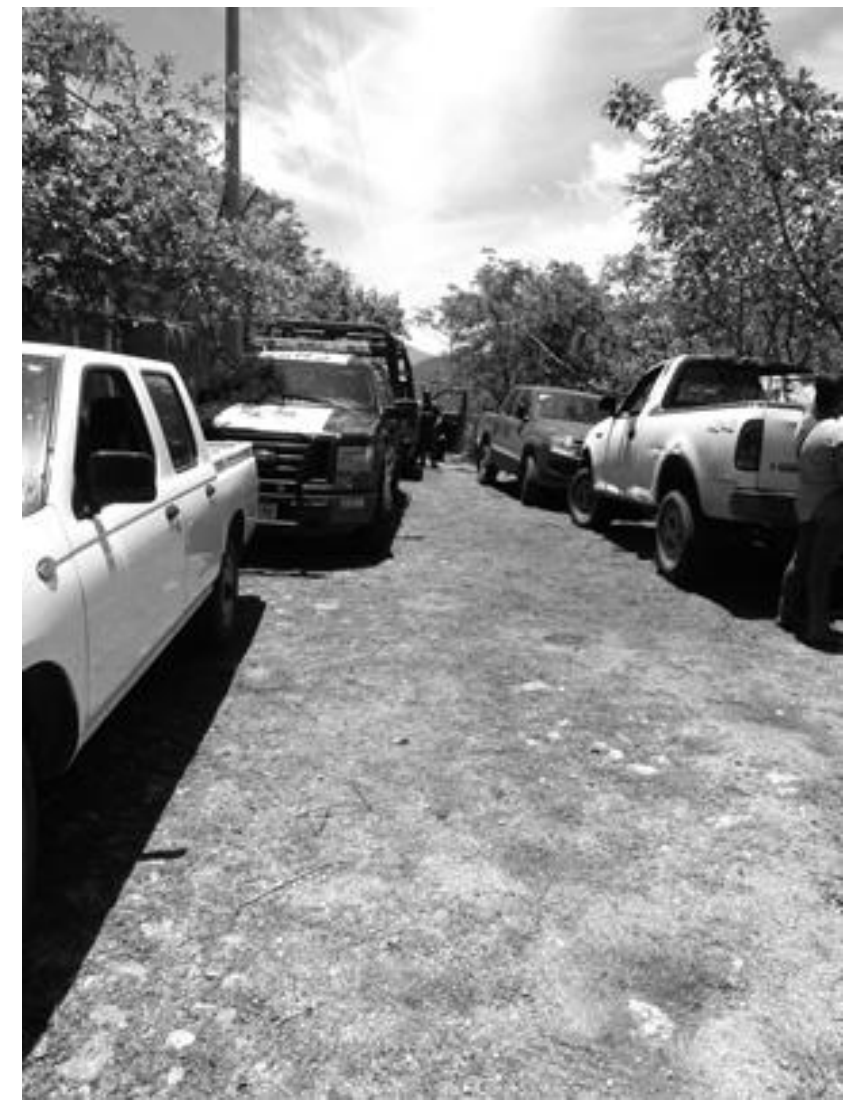

Illustration 1 - Quelques-uns des pickups qui composent le convoi pour les recherches de disparus (crédits photo : $\mathrm{S}$. Melenotte).

Le convoi dominical lourdement armé suit les pistes suggérées au groupe de chercheurs par des habitants voulant préserver leur anonymat. Cette étape de passage de l'information entre habitants d'un même village ou d'un village voisin est décisive car elle détermine la recherche du jour. Seules les familles de disparus ont accès à ces informations plus ou moins précises pour leur permettre de prospecter les zones : «des » voisins font circuler « des » informations en suivant les règles de l'omerta, racontant secrètement aux chercheurs de fosses qu'ils ont « vu » par hasard des scènes inhabituelles ou «entendu » des rumeurs, qu'ils ont repéré des objets ayant attiré leur attention, ou révélant des informations obtenus de membres de cartels qu'ils connaissent ou parce qu'ils sont eux-mêmes des «indicateurs » (halcones) pour l'un ou l'autre cartel.

Au fil des recherches, les familles se professionnalisent de facto et développent un savoir et des tech-

niques de repérage à partir de faits expérimentaux construisant des savoirs $a d$ hoc qui relèvent à la fois du chasseur, de l'archéologue, du détective et du sportif, pour retracer le parcours des criminels et lire les traces muettes de celui «qui est passé par là ». Mario Vergara, originaire de Huitzuco à côté d'Iguala, cherche son

frère Tomás. Il est devenu l'un des meilleurs «chercheurs de fosses » du pays et transmet son savoir-faire à différents collectifs. Il possède un véritable attirail empruntantà divers équipements d'escalade, d'archéologie, voire de spéléologie : des genouillères, un chapeau (chinois), un casque pour le rappel, des cordes et des harnais, une boussole, des pelles, des pioches, etc.

Mario s'entraîne physiquement pour affronter toute situation et tout climat adverse. Il a également appris plusieurs protocoles de la Croix-Rouge internationale, ainsi que l'ossature des animaux pour les distinguer des êtres humains lors des recherches de fosses clandestines.

\section{Déchiffrer la signature du crime}

Sans renoncer à retrouver en vie leurs proches disparus, les familles explorent les montagnes et développent des capacités d'observation et de déchiffrement de traces, parfois infimes, étouffées ou enterrées, qui deviennent des indices en vue d'une prospection géophysique de l'environnement. Pour illustrer le repérage de traces, voici un extrait de mon carnet de terrain du dimanche 3 juillet :

Nous partons en pickup dans la zone appelée « las antenas " (les antennes) où se trouve le principal pylône électrique de la zone. Je suis dans le pickup des familles qui rient beaucoup, les blagues fusent, les gens se taquinent. Nous arrivons les premiers et nous nous prenons en photo devant la grande antenne électrique, avant de prendre pelles et pioches pour commencer l'exploration. Les sentiers sont abrupts, mais tout le groupe se 
lance, même les personnes les plus âgées. Nous marchons au milieu des herbes hautes et touffues car la saison des pluies a débuté, ce qui ne facilite pas la recherche. Arrivé à un croisement, on hésite entre deux chemins, l'un qui monte, l'autre qui descend. Le seul indice dont nous disposons a été donné anonymement à l'un des membres du groupe, celui d'un " campement " de narcotrafiquants. Cette information a circulé de bouche à oreille, et les rumeurs disent qu'à cet endroit, il y aurait des corps, alors " des gens » ont prévenu quelqu'un du groupe qui s'y rend pour vérifier. L'information est vague et l'on ne sait pas bien si l'on est arrivé à ce campement en repérant des traces de passage humain. Après quelques minutes, on retrouve des boîtes de sardines et des cendres et l'on en déduit qu'il s'agit bien du campement. Puis le groupe se concerte pour savoir si monter ou descendre. Un peu au hasard, on décide de monter.

Toute trace de passage devient un indice : les boîtes de sardines ou les cendres laissent penser que les narcotrafiquants ont fait une halte pour faire un feu de camp (Illustration 2). Dans d'autres cas, des habits ou des bouts de corde laissent supposer que les victimes ont été attachées et déshabillées. Les familles marchent ainsi longuement sur les traces des disparus et font des arrêts quand des indices les interpellent. Alors, elles se concertent autour des traces dans la terre et élaborent à plusieurs des diagnostics :

On arrive sur une petite plaine et l'on attend les autres. Le groupe se divise en plusieurs cellules pour explorer le campement. Je suis Don Lupe et on appelle son fils après quelques minutes car on trouve un monticule de terre. La terre est soigneusement inspectée car elle possède des propriétés singulières quand il y a une fosse : ils m'expliquent que la végétation peut être plus ou moins abondante, un arbre peut avoir été coupé et la terre remuée, auquel cas, sa texture change. Elle forme soit un monticule (disparition récente), soit une dépression (disparition ancienne) car les corps prennent un certain volume puis le perdent, ce qui tasse la terre avec le temps. La couleur de la terre peut être plus sombre et la végétation plus haute.

La circulation de savoir-faire et de méthodes improvisées créé une économie judiciaire de la mort qui oblige les familles à des diagnostics proches de l'œil clinique du connaisseur et de l'amateur de tableaux analysés par Ginzburg. Elles développent une capacité descriptive et analytique pour faire émerger des caractéristiques spécifiques et des propriétés communes sur les fosses et les violences en amont. Le caractère qualitatif de cette étude improvisée renvoie au paradigme

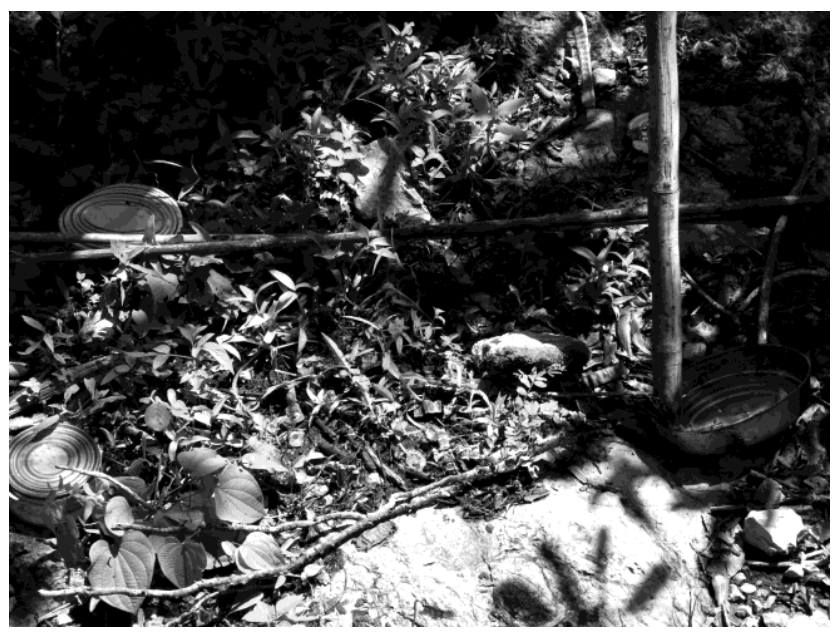

Illustration2- Des traces de passage deviennent des indices, ici des boîtes de sardines et des cendres, que les familles interprètent comme ceux d'un feu de camp de groupes criminels (crédits photo : $\mathrm{S}$. Melenotte).

indirect et indiciaire : 1' « anatomie » des fosses et de leur environnement est soigneusement analysée par les familles. Si le caractère scientifique n'est pas déterminant ici, le savoir produit par les familles sur la diversité des fosses a une efficacité directe et concrète qui leur permet de poursuivre ou non leurs recherches.

À l'inverse du paradigme galiléen, nous dit Ginzburg, le paradigme indiciaire est éminemment indirect, individuel et intuitif. En effet, la capacité des chercheurs de fosses à reconnaître et analyser les traces des violences s'est développée au fil des recherches : le savoir ainsi produit est donc indissociable de la pratique-même de la recherche, il ne s'apprend pas dans les manuels ni les protocoles. Ces formes de savoirs locaux renvoient aux gestes, aux regards, aux voix, et constituent un savoir issu de l'expérience concrète. Par exemple, les premières fosses retrouvées étaient plus retirées dans les montagnes car elles correspondaient à ce que les familles appelaient des «camps de détentions » (campos de secuestros), souvent des lieux dégagés, propres, à l'abri du soleil, qui étaient également des lieux de détente pour les narcotrafiquants. Les familles y ont également trouvé du matériel supposé servir à attacher les personnes séquestrées, tel que des cordes, des tissus, des ceintures. Près des fosses clandestines se trouvaient aussi des habits jetés, signes que quelque chose d'inhabituel avait eu lieu. L'une des découvertes les plus saisissantes pour les familles quim'ont rapporté leurs recherches concernait le degré de sophistication de certains enterrements clandestins. «Au début, il y 
avait des fosses partout », dit Xitlali. Les victimes avaient toujours des sépultures. Elles étaient souvent enterrées loin dans la montagne, en hauteur, et à un ou deux mètres de profondeur. Lors des premières recherches, alors autogérées, une première fosse avec un corps était recouverte d'une seconde fosse, découverte presque par hasard sous un énorme rocher luimême recouvert de terre. Au fil des mois, alors que les violences battaient leur plein, les familles ont trouvé des fosses plus bas dans la montagne, plus près des habitations, et les corps et les os débordaient souvent à la surface. Ces changements dans les méthodes d'ensevelissement étaient interprétés par le groupe de recherche comme le fait que les criminels étaient moins précautionneux, les corps étant ensevelis à la hâte, non loin des habitations. Les familles ont inféré les causes à partir de leurs effets : elles ont analysé ce changement en établissant une corrélation entre l'augmentation des homicides et l'ambiance de terreur qui amenait les criminels à bâcler le travail d'ensevelissement. Certaines familles ont même jugé ces signes de hâte comme étant le fait de «policiers » qui amenaient beaucoup de corps sans songer aux sépultures et encore moins aux rituels d'ensevelissement. Ces télescopages s'appuient sur ce qu'elles considèrent être des preuves et qui leur permet de créer du sens et un savoir qui circule ensuite entre les familles, sans qu'importe finalement la validation par l'État et ses organes judiciaires d'une seule et même vérité. L'emprunt de méthodes indiciaires et de bon sens suffit à gager de l'analyse ainsi établie.

Ces recherches sont soumises aux conditions climatiques. La chaleur écrasante du Guerrero alourdit les recherches, mais n'arrête aucun proche de disparu, pas même les plus anciens qui marchent des heures durant. Au contraire, la saison des pluies a pour effet direct de stopper les recherches, en raison d'averses diluviennes, mais aussi parce que les herbes hautes recouvrent les empreintes et empêchent de les discerner. Ces traces presque invisibles pour l'œil néophyte créent une ligne de démarcation plus ou moins lisible qui demande une expérience et des sens aiguisés de la part des familles pour repérer les marques des fosses incrustées dans la terre, à commencer par la vue, mais aussi par l'odeur.

Lorsqu'une fosse clandestine est repérée, quelques outils et techniques de détection servent à leur identification. On est loin des outils professionnels sophistiqués dont disposent les archéologues professionnels dans leurs pratiques d'exhumation, tels que la résistivité, où l'on plonge de l'électricité dans le sol et, selon la vitesse du retour, cela indique s'il y a du vide, si la terre a été remuée, etc. ; le géo-radar ; la magnétométrie ; le détecteur de métal. Les familles ne disposent que d'un outillage sommaire, des pelles, des pioches, des machettes et leurs sens en alerte. Quant aux experts, généralement entre 4 et 5 à chaque recherche, ils utilisent la sonde (varilla), mais les familles lui substituent la machette en leur absence. Amenée par le criminologue, la sonde est piquée dans la terre : si elle s'enfonce facilement, c'est un signe. Il faut ensuite la sortir et la sentir, ce que font souvent les familles, en se passant sonde ou la machette plantée pour analyser l'odeur (Illustration 3). Ce geste pour «sentir la mort» est une expérience sensorielle unique et subjective qui permet de distinguer une " odeur de mort » d'une odeur de terre ensevelie qui sent le moisi. La frontière olfactive est ténue, alors au moindre doute, les familles creusent à la pelle et à la pioche jusqu'à trouver des éléments permettant de confirmer qu'il s'agit, ou non, d'une fosse. Voici la suite de mon carnet de terrain :

Don Lupe plante sa machette dans la terre, puis la renifle. Entre temps, un policier fédéral nous a rejoints, avec son uniforme épais sous ce soleil tapant. Don Lupe sent la machette et trouve qu'elle sent " le moisi ", il la donne à sentir à son fils et au policier qui confirme que ça sent « la putréfaction ». Ils décident donc de creuser et demandent qu'on ramène la pelle et la pioche. Aubout d'un moment, ils s'arrêtent de creuser car la terre est remplie de grosses pierres qui empêchent de continuer plus profond, ce qui est alors considéré comme un signe qu'il ne peut y avoir de fosses dessous.

Il importe ici de souligner le savoir indiciaire et les interprétations expérimentales que forgent les familles sur les fosses, bien souvent plus audacieuses que les autorités qui les suivent, quitte à enfreindre bien des protocoles et à courir le risque de ce que les experts appellent la «pollution de la preuve» (contaminación de evidencia) au moment de planter la machette dans la terre ou de creuser à la pioche.

Sur le chemin du retour, si elles reviennent bredouilles, les familles collectent avec les mêmes machettes, les plantes médicinales locales qu'elles échangent, dont elles vantent les vertus thérapeutiques, et qu'elles ramènent chez elles à défautd'avoir trouvé des morts (Illustration 4). Ce soin symbolique du cœur vient clore la journée de marche qui aura alors pris davantage une allure d'expédition dominicale sous la chaleur écrasante du Guerrero. 


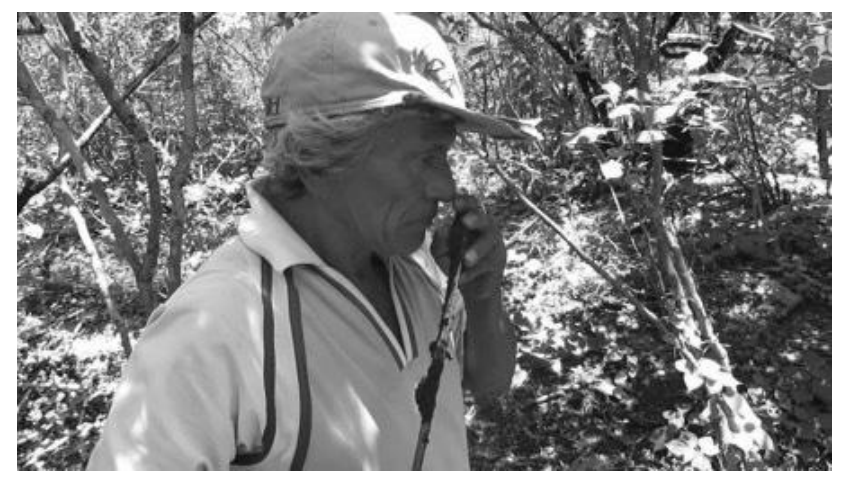

Illustration 3 - Don Lupe sent sa machette après l'avoir plantée dans la terre afin d'interpréter l'odeur avant de creuser davantage ou non (crédits photo: $\mathrm{S}$. Melenotte).

\section{- Lire le cadavre}

\section{Le tournant médico-légal au Mexique}

«Parler du cadavre » [Courtois, 2015] suppose que le cadavre parle. L'étude du corps alliée à celle de la mort resurgit ces dernières années autour du « tournant médico-légal » [Anstett et Dreyfus, 2015] qui interroge l'irruption de nouvelles technologies et lance de nouveaux enjeux de biopouvoir [Anstett, 2013]. L'expertise médico-légale suscite un regain d'intérêt en histoire, en anthropologie sociale, et en droit, dans des disciplines qui s'emparent de cette science et en font un nouvel objet d'étude [Anstett et Dreyfus, 2015]. Depuis le dernier quart de siècle, l'injonction à retrouver des traces matérielles des violences passées traduit un tournant quantitatif et qualitatif majeur, lié à l'innovation technologique et à l'impératif de faire apparaître les morts pour mieux connaître le passé. L'ex-Yougoslavie a été le premier cas traité par un Tribunal pénal international (TPIY). Depuis, les exhumations de fosses se sont multipliées, au Cambodge, au Rwanda, en Espagne, au Guatemala, etc. Même si le phénomène est variable d'un pays à l'autre, de nombreuses fosses et exhumations " à l'âge des droits humains » [Ferrándiz et Robben, 2015] révèlent que les cadavres sont non seulement porteurs de droits, mais surtout qu'ils détiennent la capacité de ré-humaniser des « restes sans noms et des noms sans corps » [Huffschmid, 2015]. L'étape d'identification est donc cruciale dans le cas mexicain car elle construit les indices probatoires à venir. Ces indices, aujourd'hui en cours de fabrication, permettront dans le futur de construire des récits appartenant à la sphère judiciaire,

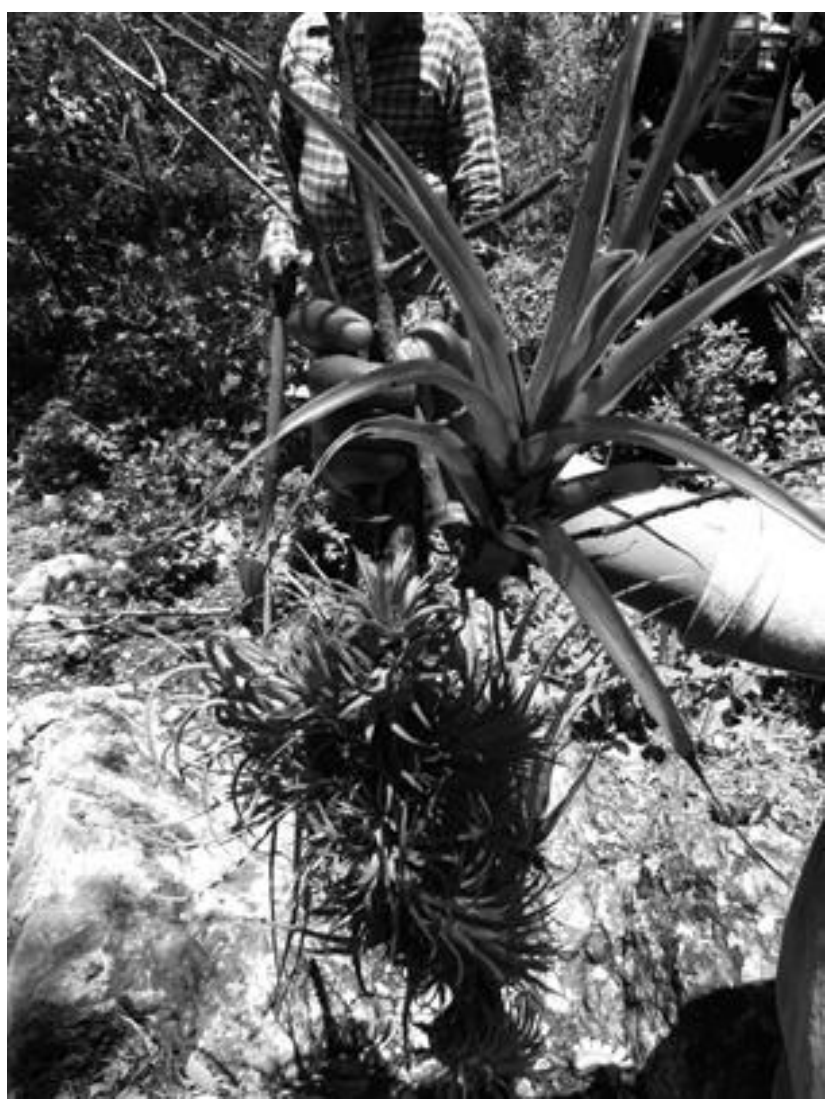

Illustration 4 -Quelques plantes médicinales récoltées en fin de journée comme un soin du cœur (crédits photo: $\mathrm{S}$. Melenotte).

pour servir des techniques d'accusation ou de défense. Les traces laissées sur les corps deviendront des preuves matérielles qu'il faudra interpréter dans les espaces de procès, afin de comprendre le degré d'intentionnalité, l'organisation politique et criminelle en amont de ces violences, leur caractère massif, orchestré ou planifié, leur ancrage local, le type d'armes et les formes ritualisées de mise à mort, etc.

$\mathrm{Au}$ Mexique, l'arrivée de la technologie génétique depuis les années 2000 a transformé la possibilité de déterminer l'ADN des particules osseuses. Avant, on disposait de radiographies, de fiches odontologiques, de dossiers hospitaliers, quand ces documents étaient établis, ce qui n'était pas nécessairement le cas dans les zones rurales et marginales. Avec la génétique, on a pu procéder à des croisements massifs de données sans avoir à passer nécessairement par une hypothèse d'identité au préalable. Des bases de données génétiques peuvent se constituer sur de simples prises de 
sang, mais selon des règles précises en fonction du lien de parenté. En Argentine, on a pu ainsi reconstituer le profil génétique de plus de 4200 personnes. Cela représente moins de la moitié des disparus, estimés entre 10 à 12000 lors de la dictature militaire, mais il s'agit d'une réussite non-négligeable, plus de la moitié d'entre eux ayant été jetés dans le Rio de la Plata et étant donc inaccessibles.

Aujourd'hui, on prête aux experts légistes un pouvoir divinatoire, au sens de Ginzburg, c'est-à-dire qui se tourne à la fois vers le passé et vers le futur. Ils auraient cette capacité de déchiffrer des os et de reconstituer un corps démembré, offrant ainsi la possibilité de réintégrer le disparu au monde social, et même de reconstruire la scène du crime et comprendre des modèles et des logiques de terreur en amont. Les experts légistes disposent donc d'un pouvoir tout à fait singulier, celui de «nommer» les actes passés. Dans le cas des disparus en Argentine, la célèbre Équipe argentine d'anthropologie médicale (Equipo Argentino de Antropología Forense, EAAF) a réussi, à partir de quelques os retrouvés sur les berges du Rio de la Plata, à retrouver l'identité des disparus et à reconstituer ce qu'il s'était passé : les fragments d'os ont révélé que le corps était tombé d'une hauteur très élevée et, en croisant les données, il s'est avéré que tel corps avait tel nom, Pedro, Cristina, etc., et qu'ils avaient été victimes des fameux «vols de la mort» [Huffschmid, 2015]. De même, le groupe indépendant guatémaltèque qu'a suivi Victoria Sanford [2003] révèle, avec les témoignages recueillis par la Commission d'éclaircissement historique, les méthodes d'annihilation sur les corps, les techniques de torture des corps d'élite guatémaltèques, les kaibiles. L'équipe guatémaltèque a pu récupérer 6500 corps, la plupart lors d'exhumations dans les communautés mayas, où plus de la moitié ont été identifiés grâce aux analyses génétiques. L'interprétation des experts a ensuite joué un rôle décisif lors du procès de l'ancien dictateur Efraín Ríos Montt : quand, pour la première fois, un ancien président se trouvait devant un tribunal national pour délit de génocide, l'équipe médico-légale et d'autres organismes ont apporté plus de 60 expertises. Les fragments retrouvés dévoileraient donc le destin d'un être humain mais aussi d'une nation tout entière, grâce aux experts qui détiennent les savoirs leur conférant une autorité et une légitimité opérantes. Selon Huffschmid, il s'agit donc, bien plus qu'une simple technique relevant de la criminologie, d'un rôle réel pour le travail de mémoire et de fonction sociale dans les violences extrêmes : ils créent la jonction entre des os sans noms (restes anonymes) et des noms sans corps (disparus) et rendent leur humanité à des restes de corps enterrés clandestinement.

$\mathrm{Au}$ Mexique, le déchiffrement et l'interprétation des familles confrontent ces experts à un conflit de valeur absolument déterminant qui sépare le savoir produit par les familles et celui du «tournantmédicolégal ». Il renseigne sur l'évolution du paradigme indiciaire avec l'introduction de nouvelles technologies, ainsi que sur le rôle des experts dans l'identification des corps, qui n'interviennent qu'une fois la fosse trouvée. L'attente placée dans ces nouvelles technologies peut être immense. Pour Ernesto Schwartz Marín et Samuel Taylor-Alexander [2013], qui suivent les modes de rationalité émergents dans l'Institut mexicain de Génétique (INMEGEN) l'enjeu est bien plus considérable encore que le développement d'une « nouvelle » technologie. La « souveraineté génomique » crée des imaginaires nouveaux faisant de la génétique une ressource politique permettant la projection et qu'ils qualifient de « bioprophétie » : selon eux, les banques de données promettent de raconter le futur et participent de l'ambition nationale du Mexique d'obtenir le développement « dernier cri » et « high tech » pour parachever ainsi sa modernisation ${ }^{9}$. L'histoire et les technologies feraient ainsi la fierté nationale, à travers des projets politiques qui rejouent les droits et les responsabilités des citoyens. Les auteurs ne parlent pas directement des disparitions et s'attachent plutôt à montrer l'usage de cet Institut national génomique pour prévenir les maladies en connaissant mieux le « génome mexicain », ce qui n'est pas sans lien avec de fortes questions identitaires et raciales liées au métissage. Mais en tant que nouvelle technologie, les tests ADN utilisés auprès des familles de disparus participent de cette "souveraineté génomique » qui contribue à la « santé » de la nation. La métaphore est d'ailleurs filée par les familles de disparus elles-mêmes qui mentionnent constamment la «blessure ouverte» (herida abierta) que représente la disparition de leurs proches. Cette analogie avec le corps malade qu'est aujourd'hui la nation mexicaine traduit également le décalage entre cette communauté génomique imaginée et les pratiques et expériences quotidiennes de familles inconsolables.

Les tests ADN à l'épreuve des familles de disparus

Lors des recherches de fosses clandestines, une équipe rotative d'experts (criminologue, odontologue, 
photographe médico-légal, vidéaste médico-légal, anthropologue physique) intervient dans un deuxième temps pour aider à identifier des fosses clandestines avant les exhumations. En 2016, la situation encore nouvelle de recherche de fosses par les familles et de traitement de morts de masse dépassait visiblement le parquet fédéral qui ne disposait que de 38 experts officiels travaillant pour lui sur l'ensemble du territoire national. Ces derniers se relayaient chaque semaineet il était rare de retrouver la même équipe d'une recherche de fosses à une autre. Parfois, une unité canine les accompagnait. Les chiens aidaient également à localiser une fosse, en grattant avec frénésie quand ils sentaient une odeur suspecte. Mais leur rôle était minime lors de ces recherches de fosses clandestines dirigées par les familles les plus actives. L'action des experts était différée à la morgue ou pendant les exhumations pour identifier les cadavres, voire les méthodes de mises à mort.

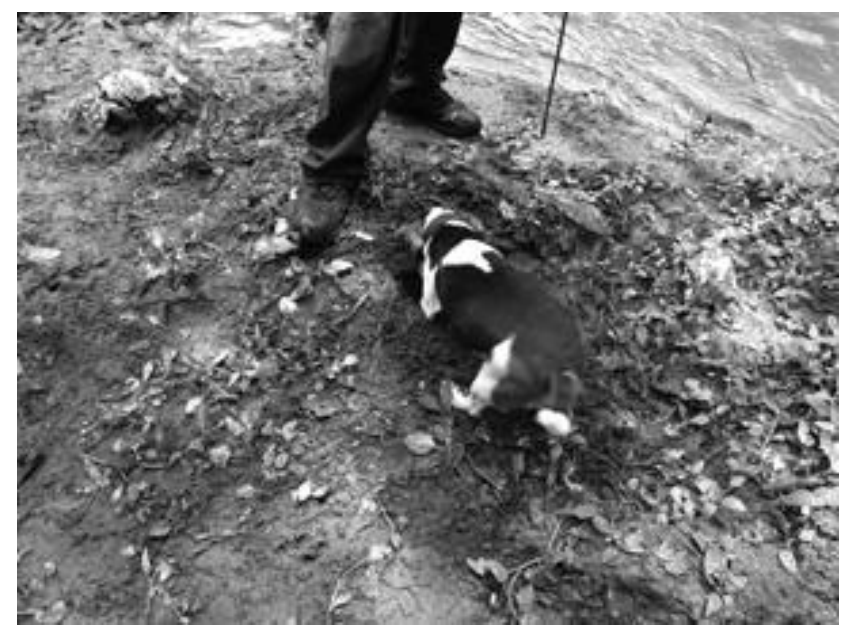

Illustration 5 - Un chien de l'unité canine du procureur gratte la terre quand il détecte une odeur suspecte (crédits photo : S. Melenotte).

Une fois les cadavres retrouvés, prélever des échantillons sanguins et réaliser des tests ADN pose une série de problèmes. Le caractère massif des corps morts qui dérangent aboutit à la saturation des espaces traditionnels de la mort tels que les morgues et les cimetières ou panthéons. Les services médico-légaux (SEMEFO) d'Iguala et de Chilpancingo - c'est aussi le cas dans plusieurs états du Mexique - débordent littéralement de cadavres retrouvés qui restent des semaines, voire des mois, sans être identifiés, par manque de moyens. Rien qu'à Iguala, en 2016, sur les 150 cadavres retrouvés, seuls 28 corps avaient été identifiés par des experts débordés par une situation où les étapes d'identification et d'enquêtes piétinent, malgré la pression des familles. Les chances d'identification par les moyens classiques de l'autopsie médicolégale décroissant à mesure que les délais augmentent, la décomposition progressive effaçant progressivement les traces du crime visibles sur le corps, les tests ADN s'imposent. Pourtant, l'introduction de ces nouvelles technologies censées déchiffrer les corps anonymes retrouvés pose rapidement la question de l'inégalité de l'accès à ces tests et échantillons onéreux pour des familles issues des zones rurales les plus marginalisées du pays. Les collectifs et associations les mieux organisés ont pu bénéficier, avant l'action des autorités judiciaires, de tests ADN offerts par des groupes indépendants ou des associations et procéder à des prélèvements sur les proches ayant enregistré les disparus. À Iguala par exemple, une fois les 350 plaintes déposées, les familles de disparus ont pu réaliser les premiers tests ADN avec le « Groupe médico-légal citoyen de sciences » qui a voulu créer une banque de données au service de la société civile. Ce groupe a «offert» 500 tests ADN aux familles de disparus qu'ils ont ensuite envoyés à Birham, en Angleterre. Après des mois d'attente, les résultats ont finalement été envoyés aux familles, mais sans être corroborés avecles cadavres anonymes retrouvés et stockés dans les morgues, rendant inutile la coûteuse opération.

Jusqu'à aujourd'hui, et malgré l'adoption de la «Loi sur la disparition forcée » et le changement de gouvernement, l'État mexicain n'a pas encore de méthodologie d'investigation pour affronter sérieusement les disparitions massives. Un protocole de recherche des disparus devait être mis en place en 2013 sur la base des standards internationaux, mais ce protocole n'existait toujours pas quand je débutais mes recherches en 2016. Les procureurs espéraient en voir naître un en décembre 2015, en s'inspirant du modèle «AM/PM » (Ante Mortem/Post Mortem) appliqué par la Croix rouge internationale. Ce protocole s'appuie sur un logiciel spécialisé conçu pour mettre en évidence les correspondances entre deux types de données, les profils de familles de personnes disparues en intégrant leurs données ADN, et ceux des corps retrouvés non identifiés par les familles. En 2014, moins de la moitié des États fédérés avait signé la convention pour l'intégration des banques d'information génétique, pourtant indispensables pour que tout le système fonctionne. Depuis, la Loi sur la disparition forcée a été adoptée, obligeant désormais les entités fédérées à signer les conventions 
nécessaires. Mais, même si la Loi implique de suivre un protocole national pour agir efficacement dès les 72 heures suivant les faits, celui-ci peine à être appliqué. Une base de données existe déjà au niveau des Etats fédérés du pays et est régulièrement actualisée avec les éléments nouveaux qui surgissent des familles ou des experts, ce qu'on appelle le processus d'homologation. Désormais, le défi majeur est de créer une base de données publique avec les caractéristiques des corps retrouvés et non-identifiés, pour avancer pas à pas vers la construction d'une seule base de données, homologuée, où les critères seraient croisés.

Le «Mouvement pour nos disparus » dans le Morelos a proposé très tôt aussi, avec le soutien du poète Javier Sicilia et l'université autonome, de construire une base de données au niveau national à partir du travail d'organisation et d'analyse des familles, pour rendre publics les profils des corps et dresser des profils de disparus, dont on ne sait finalement pas grand-chose. En s'alignant sur la méthode internationale, il serait possible d'établirune base de données ante mortem, m'explique Roberto Villanueva qui dirige le programme d'attention aux victimes à l'université autonome du Morelos. À partir d'entretiens avec les familles, il est possible de déterminer le sexe, l'âge, la taille, les habits, les caractéristiques identitaires (fractures, cicatrices, couleurs de cheveux, longueur des cheveux, grains de beauté, coupure, ou tout signe distinctif), et tenter d'identifier les causes de la mort, le lieu, etc. En procédant de la sorte, le test ADN n'est finalement qu'une ultime option : il ne viendrait que confirmer les signes distinctifs que les famillesavaientdéjàreconnus parles méthodes d'identification anciennes. En2016,j'observais pourtant la promotion des tests ADN par les autorités pour retrouver les disparus lors des réunions à l'église de San Gerardoà Iguala. Des ateliers avaientété mis en place pour sensibiliser les familles et les pousser à faire le test rapide : les avocats du Centre national des droits humains (CNDH) et les autorités expliquaient à tour de rôle les liens de parenté nécessaires pour que le test ADN soit efficace. Cette campagne devait convaincre les familles qui refusaient de donner leur sang, soit par ignorance de la procédure, soit par peur, soit encore pour ne pas rouvrir la plaie de la disparition, ou enfin par résignation. Souvent aussi, les familles ont dû réaliser plusieurs tests ADN pour plusieurs organismes : ONG, associations et institutions étatiques. La démultiplication des tests n'a pas pourautantsignifiél'identification, lesrésultatsdes tests ADN ne pouvant, là encore, être corroborés par manque de croisement des données.
Les tests ADN peuvent également être contestés par les familles qui accumulent beaucoup de méfiance à l'égard des autorités et des institutions mexicaines. Par exemple, à Chilpancingo, la capitale de l'État du Guerrero, j'ai rencontré dans un centre de défense des droits humains un père de famille qui attendait une contre-expertise réalisée par l'équipe argentine de médecins légistes indépendants. Il avait eu recours à celle-ci après avoir reçu un rapport d'autopsie des autorités et un test ADN qu'il contestait car il ne reconnaissait pas celui que l'on voulait faire passer pour son fils. Il a mené luimême sonenquêteen s' informant surles transformations possibles d'un corps eten ressortaitformel : certains traits ne peuvent changer ni être altérés, comme l'espace entre les yeux ou la forme des oreilles. Et, me disait-il, il manquait dans le rapport d'autopsie des caractéristiques, comme une cicatrice sur le genou. Autant d'indices qui l'amenaient à ne pas croire le test $\mathrm{ADN}$ officiel. Ce jourlà, la contre-expertise du groupe argentin est venue confirmer l'expertise officielle : il s'agissait bien de son fils. Au-delà de son « déni de mort », ce père de disparu, en me décrivant minutieusement les caractéristiques uniques de son fils, témoignait avant tout de la méfiance envers les institutions et envers les nouvelles technologies qui oubliaient chacune des traces pourtant capitales à ses yeux devenus, un instant, experts. Mais surtout, il développait un savoir concret lié au bon sens.

Bien que censés représenter un effort de rationalisation et de modernisation de la vérité, les tests $\mathrm{ADN}$ sont donc loin de créer un consensus. La démultiplication des tests ADN par différentes institutions ou entreprises privées qui démarchent auprès des familles de disparus, sans jamais réunir les résultats, ni les remettre aux familles, interrogent ce recours à la science supposée faciliter la vérité. Loin d'être acquise et d'aller de soi, cette économie judiciaire et politique dans l'emploi de la génétique pour rationaliser les violences passées n'établit pas la façon dont ces victimes ont été tuées, l'ethnie ou l'organisation à laquelleils appartenaient, la méthode par laquelleils ont été tués, en reconstituant les scènes du crime. Or, comme le rappelait Élisabeth Claverie à propos des corps retrouvés de personnes disparues pendant la guerre en Bosnie, les pratiques d'identification devraient permettre in fine de répondre à la question « comment ces personnes se sont-elles retrouvées, mortes, dans des fosses », qu'elles soient communes ou clandestines ?Quels liens existait-il entre elles? L'enjeu est de taille car il permettrait de qualifier les violences et surtout de distinguer les violences politiques, celles des forces militaires, policières, des violences criminelles produites par les narcotrafiquants. 


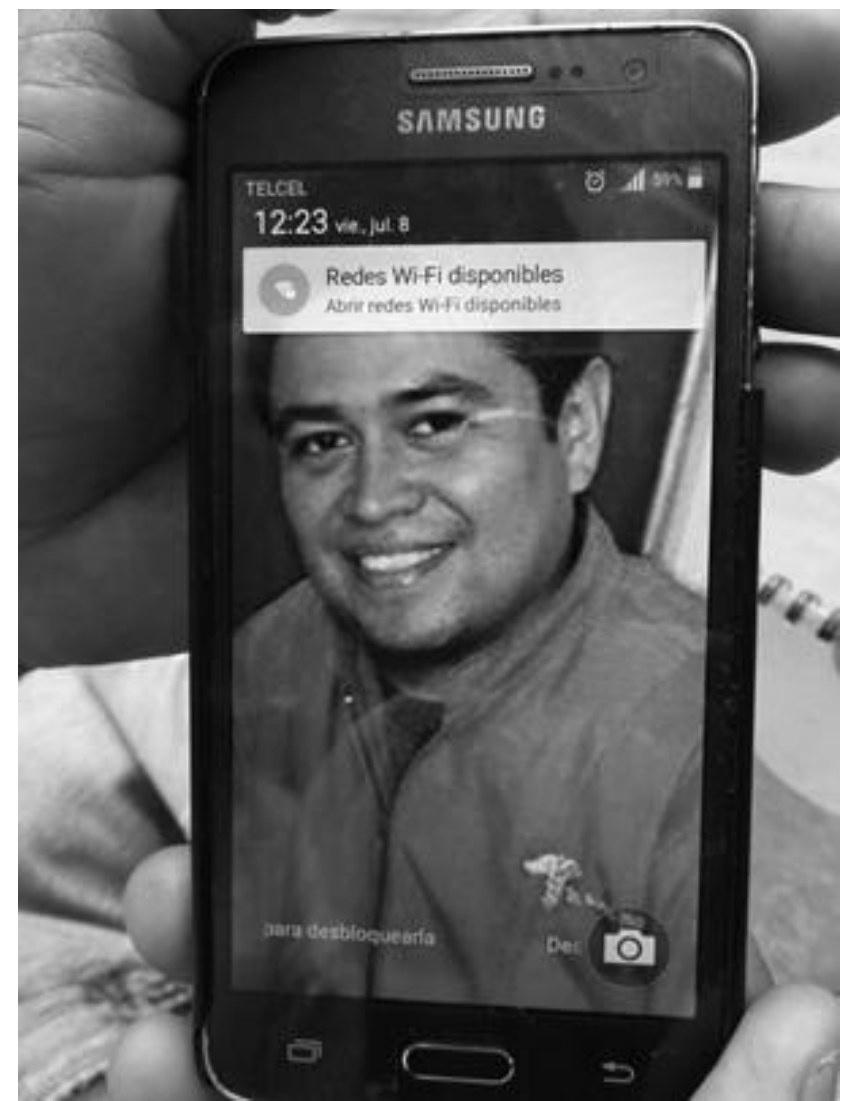

Illustration 6 - Photographie d'un disparu sur le téléphone de son père qui la compare à celle de l'autopsie pour contrer le résultat du test ADN (crédits photo : S. Melenotte).

Enfin, le problème majeur aujourd'hui au Mexique réside certainement dans le manque de justice adossée à ces nouvelles technologies : la fonction pénale de ces identifications est limitée, voire inexistante. L'identification des corps, quand elle a lieu, n'est presque jamais suivie d'enquêtes approfondies. Quand je débutais mes nouvelles recherches, une Unité spécialisée pour la recherche de personnes disparues existait depuis 2013. En octobre 2015, elle était devenue un Bureau du procureur spécial pour la recherche de personnes disparues (Fiscalía para Búsqueda de Personas Desaparecidas), affilié alors au parquet fédéral (PGR). En 2016, lorsque je leur demandais des chiffres, seuls 846 dossiers avaient étés traités et 12 membresétaient en charge de l'enquête. De même, la coordination entre ce bureau fédéral et les instances fédérées généralement en charge des enquêtes n'était pas assurée, créant des doublons, voire des tensions entre les institutions, qui s'ajoutaient au dédale bureaucratique des proches de disparus. Ce sous-effectif flagrant de personnel a ensuite été pallié et l'essor des professions légistes au Mexique vise depuis à combler ce manque. Aujourd'hui, la Commission nationale de recherche de disparus a été créée et le personnel compétent en cours de formation, mais les coupes budgétaires interrogent l'avenir de ces nouvelles initiatives censées résoudre la «crise légiste » au Mexique.

\section{- Conclusion}

Ces descriptions ethnographiques mettent en lumière la présence systématique de nombreux acteurs institutionnels, des experts scientifiques, des policiers, des militaires, des associations et défenseurs des droits humains, et les familles de disparus, qui gravitent autour des fosses clandestines et des corps retrouvés. La marche et l'effort fourni par les familles de disparus font de ces recherches de nouveaux rituels dont la dimension sacrée réintroduit du sens et fait communiquer les vivants avec les morts et les disparus. La performance physique est entière et les sens, surtout la vue et l'odorat, sont particulièrement mobilisés durant ces recherches. La découverte d'une fosse clandestine ou d'un cadavre est un trésor, me disent souvent les familles de disparus. Elle permet l'apaisement du deuil même si elle ravive sur le moment la douleur de la disparition de l'être cher. Parfois aussi, les recherches, surtout quand elles n'aboutissent à aucune découverte de fosses, peuvent prendre des airs d'expédition dominicale. À défaut de retrouver des disparus, les familles auront passé la journée avec des membres d'une petite communauté que la douleur d'un proche disparu réunit et qui, non sans force symbolique, finissent par revenir les mains chargées de plantes et herbes médicinales. Mais surtout, elles confrontent familles, autorités et experts à un conflit de valeur absolument déterminant. Les alliances, les tensions et les rapports de force se nouent et se dénouent autour de traces laissées par les violences, les acteurs s'opposent et entrent en concurrence dans l'établissement d'une seule et même " vérité » sur ce qui s'est « réellement » passé. Toutes ces traces sont, pour les familles qui cherchent leurs disparus, autant d'indices qui serviront de preuves dans des enquêtes et des procès futurs. Le paradigme indiciaire développé dans un contexte judiciaire rend donc les traces retrouvées déterminantes pour qualifier des 
violences politiques et criminelles voire, pour réécrire à long terme le récit national mexicain.

Contre toute attente, cette concurrence autour de la vérité sur les violences passées qu'il faut établir n'est finalement pas résolue par l'introduction de la génétique. Au contraire, les disputes autour de l'attestation et de la validité delascienceetdes nouvelles connaissances ainsi produites invitent l'anthropologue à intégrer les «écarts épistémologiques » [Makaremi, 2017] entre les récits, les usages sociaux et les significations de toutes ces traces pour les familles, les experts et les autorités. Les traces des violences deviennent des signes qui permettent aux différents acteurs de tenter une remise en ordre, au moins symboliquement, du chaos. Suivreles traces des disparus revient à partir à la recherche de « traces infinitésimales » dans la nature ou sur les cadavresretrouvés. Maiscestracesn'ontpas desens «en soi » : c'est leur mise en contexte dans des rapports sociaux et les tentatives pourdéchiffrer etinterpréterces traces qui en font des signes. Les enjeux pour suivre les traces des disparus sont donc aussi bien politiques qu'herméneutiques, voire épistémologiques. Le lien entre la chasse et la génétique explique alors comment l'observation de données expérimentales permet de remonter à une réalité éminemment complexe, le passage à l'acte violent, qui n'est finalement jamais totalement observable directement. Les familles pistent en premier les disparus et l'anthropologue à son toursuit les familles de disparus et observe les pratiques de tous les acteurs qui convergent dans les paysages de la mort. Dans un tel contexte, l'anthropologie s'attèle finalement à raconter et décrire les usages sociaux et culturels générés par les traces des violences, pour « déchiffrer » et « lire » la société contemporaine, en introduisant du sens dans ce chaos manifeste.

\section{Notes}

1. Le Mexique est un État fédéral composé de 32 États. La gouvernance se gère à trois niveaux : municipal, régional (dans chaque État) et fédéral. L'Etat du Guerrero est composé de 81 municipalités (municipios) et Iguala de la Independencia est la troisième ville de l'État la plus peuplée après Chilpancingo et Acapulco.

2. Ginzburg démontre l'origine commune de ce que l'on considère aujourd'hui comme des pseudosciences, la divination et la physiognomonie, et des sciences comme le droit et la médecine, la perméabilité de termes techniques tirés du lexique juridique se retrouvant dans des traités divinatoires à partir d'éléments de physiognomonie et de sémiotique médicale. Ces formes de savoir héritées de l'ancienne Mésopotamie sont également imprégnées du paradigme indiciaire issu de la reconstruction de traces, de symptômes, d'indices. Inversement, la médecine s'appuiesur des traités divinatoirespourétablirdes diagnostics.

3. Agustín de Iturbide y a proclamé le Plan d'Iguala en 1821 qui reconnaît l'indépendance du Mexique vis-à-vis de l'Espagne. C'est là aussi qu'a été confectionné le drapeau mexicain qui flotte aujourd'hui encore sur le flanc des montagnes à l'arrivée dans la ville. Iguala est également mentionnée dans le Document d'indépendance du Mexique ainsi que dans l'hymne national.
4. Auparavant, les routes étaient sous contrôle duCarteldes BeltranLeyva, puis des « Guerreros Unidos » (Guerriers Unis) (au moment d'Ayotzinapa) et la municipalité d'Iguala est devenue une route de transit obligée pour les Cartels. Aujourd'hui, plusieurs micro-cartels tels que «Los Bélicos » (les Guerriers), «Las Ardillas » (les Écureuils) et «Los Rojos » (les Rouges) se disputent « la plaza » (la place).

5. Jusqu'à cette date, une loi tenait compte des «personnes non-localisées ». Par disparition forcée, l'État reconnaît désormais le rôle d'agents de l'Etat à tous ses niveaux dans les disparitions. Cette avancée juridique est pourtant un retard sur la réalité : le Guerrero cumule une histoire longue de disparitions pour motif politique, avec la présence d'importantes guérillas dans les années 1970, dont celles de Lucio Cabañas et Génaro Vazquez, chassés et disparus au moment de la «Guerra sucia » [Guerre sale].

6. Créée par la population en 2011 pour se protéger de la violence des cartels et de la corruption des autorités, l'UPOEG (Unión de los Pueblos y Organizaciones en el Estado de Guerrero) est, avec la Coordinadora Regional de Autoridades Comunitarias - Policía Comunitaria (CRAC-PC), l'une des principales réponses citoyennes armées dans l'Etat du Guerrero, située surtout dans la Montaña et la Costa Chica, et présente dans la zone de production de café auprès d'une douzaine de municipalités [municipios] sur les 77 de toutl'État.L'unde ses principaux représentants, Miguel Angel Jiménez, a été assassiné en août 2015 dans une embuscade.
7. Le parquet fédéral (Procuraduría General de la República, PGR) est l'organe du pouvoir exécutif fédéral au Mexique qui a à sa charge les investigations et poursuites pour des délits d'ordre fédéral. La PGR préside le ministère public de la fédération et ses organes auxiliaires que sont la police et les experts judiciaires et légistes.

8. Avant la Loi sur la disparition forcée de 2017, la gestion des dossiers des personnes «non-localisées » correspondaient aux instances fédérées en charge des enquêtes (procuradurías et fiscalias). En 2016, lors de mon premier terrain, seuls 966 cas relevaient du droit fédéral et étaient pris en charge par le parquet fédéral, les 28937 autres cas relevaient des bureaux spéciaux au niveau fédéré. Ce n'est qu'à la fin 2017 qu'un plan national se met en place. Le dynamisme des familles d'Iguala a obligé le parquet fédéral à prendre en charge les recherches de fosses bien avant le vote de la loi.

9. Leur argument va loin : le futur se modèle par des prédictions économiques qui fournissent un socle rationnel à celui-ci et permettent l'action. Selon eux, ces prédictions aident à fournir un lien entre la santé des individus, la population «métisse », et le futur de la nation. La médecine génomique est censée contrer la dégradation biologique, économique, politique, et pas uniquement les corps malades. Elle doit substituer au Mexique faible une nation forte. 


\section{| Références bibliographiques}

ANSTETT Élisabeth, 2013, « Des cadavres en masse : sociétés et sciences sociales face à l'impensé. Techniques et culture », Techniques et cultures, 60 [Hervé Guy, Agnès Jeanjean, Anne Richier (dir.), Le Cadavre en procès] : 126-143.

ANSTETT Élisabeth et Jean-Marc DREYFUS, 2012, Cadavres impensables, cadavres impensés. Approches méthodologiques du traitement des corps dans les violences de masse et les génocides, Paris, Éditions Petra.

ANSTETT Élisabeth et Jean-Marc DREYFUS, 2015, Human remains and identification. Mass violence, genocide, and the "forensic turn", Manchester, Manchester University Press.

AnSTETT Élisabeth et Luba JuRGENSON, 2009, Le Goulag en héritage. Pour une anthropologie de la trace, Paris, Pétra.

CLAVERIE Élisabeth, 2011, « Réapparaître. Retrouver les corps des personnes disparues pendant la guerre en Bosnie », Raisons politiques, 41 (1) : 13-31.

Courtols Martine, 2015, « Parler du cadavre », Communications, 97 (2) : 17-27.

CYR Rachel E., 2014, « The "Forensic Landscapes” of Srebrenica », Култура/Culture, 5 : 81-92.

DIDI-HuBERMAN Georges, 2004, Images malgré tout, Paris, Les Éditions de Minuit, coll. « Paradoxe ».

DULONG Renaud, 1998, Le Témoin oculaire. Les conditions sociales de l'attestation personnelle, Paris, Éditions de l'EHESS.
ESCALANTE Fernando, 2011, « Homicidios 2008-2009 : la muerte tiene permiso », Nexos, janvier 2011 [http://www.fernandoescalante.net/el-homicidio-en-mexico-nuevosnumeros/, consulté le 18 mars 2015].

FERRÁNDIZ Francisco et Antonius C.G. RoBBEN, 2015, Necropolitics. Mass Graves and Exhumations in the Age of Human Rights, Philadelphia, University of Pennsylvania Press.

GATTI Gabriel, 2017, Desapariciones. Usos locales, circulaciones globales, Colombia, Siglo del Hombre Editores/Universidad de los Andes.

GinZBURG Carlo, 1980, « Signes, traces, pistes. Racines d'un paradigme de l'indice », Le Débat, 6 : 3-44.

HuFFSCHMID Anne, 2015, «Huesos y humanidad : Antropología forense y su poder constituyente ante la desaparición forzada », Athenea digital, 15 (3) : 195-214.

MAKAREMI Chowra, 2017, " "États d'urgence ethnographiques". Approches empiriques de la violence politique ", Cultures \& Conflits, 103-104 [Chiara Calzoialo, Pamela Colombo et Chowra Makaremi (dir.), Ethnographies politiques de la violence ] : 15-34.

Musset Alain, 2015, Le Mexique : "Que sais-je? », 1666, Paris, Puf.

TAYlOR-AlEXANDER Samuel et Ernesto SchWARTZ MARÍn, 2013, «Bioprophecy and the politics of the present: notes on the establishment of Mexico's national genomics institute (INMEGEN) », New Genetics and Society, 32 (4) :333-349.

SANFORD Victoria, 2003, Buried Secrets Truth and Human Rights in Guatemala, New York, Palgrave Macmillan.

SÉMELIN Jacques, 2005, Purifier et détruire, : Usages politiques des massacres et génocides, Paris, Éditions du Seuil. 
